\title{
Electron-beam diagnostic for space-charge measurement of an ion beam
}

\author{
Prabir K. Roy, a) Simon S. Yu, Enrique Henestroza, Shmuel Eylon, Derek B. Shuman, \\ Jozsef Ludvig,, Frank M. Bieniosek, William L. Waldron, Wayne G. Greenway, \\ David L. Vanecek, Ryan Hannink, ${ }^{\mathrm{c})}$ and Monserrat Amezcua \\ Lawrence Berkeley National Laboratory, University of California, 1 Cyclotron Road, Berkeley, \\ California 94720
}

(Received 17 November 2004; accepted 19 November 2004; published online 5 January 2005)

\begin{abstract}
A nonperturbing electron-beam diagnostic system for measuring the charge distribution of an ion beam is developed for heavy ion fusion beam physics studies. Conventional diagnostics require temporary insertion of sensors into the beam, but such diagnostics stop the beam, or significantly alter its properties. In this diagnostic a low energy, low current electron beam is swept transversely across the ion beam; the measured electron-beam deflection is used to infer the charge density profile of the ion beam. The initial application of this diagnostic is to the neutralized transport experiment (NTX), which is exploring the physics of space-charge-dominated beam focusing onto a small spot using a neutralizing plasma. Design and development of this diagnostic and performance with the NTX ion beamline is presented. (C) 2005 American Institute of Physics.

[DOI: $10.1063 / 1.1847392]$
\end{abstract}

\section{INTRODUCTION}

The heavy ion fusion (HIF) program ${ }^{1}$ includes a series of experiments $^{2-4}$ designed to test concepts for acceleratorbased inertial fusion drivers as well as high energy density physics. One such experiment, the neutralized transport experiment $^{2}$ (NTX), investigates the use of beam neutralization techniques to mitigate space-charge-induced beam blow-up as the beam travels the last few meters to the target. NTX utilizes a $\mathrm{K}^{+}$beam ${ }^{5,6}$ having a large radius at the entrance to the neutralization section, and it is this beam that is to be characterized. Measurement of the charge distribution and the phase space of an ion beam using conventional intercepting diagnostics such as a Faraday cup, slit cup, wire scanner, pepperpot, ${ }^{7,8}$ or scintillator often completely disrupts the ion beam itself. This is presently unavoidable for phase space measurements, though total beam charge can be measured nonperturbatively using a Rogowski coil or other similar inductive probes located around the beam. Such devices cannot quantify the transverse cross-sectional charge distribution in the beam. The diagnostic system presented here is designed to nonperturbatively quantify the transverse cross sectional charge distribution, assuming the beam is "long" and slowly changing in the longitudinal direction. Recently a few attempts ${ }^{9-11}$ have been made to use an electronbeam probe for monitoring the longitudinal charge distribution of small diameter relativistic electron bunches where magnetic fields predominate. These diagnostics are not suitable for measurement of large diameter nonrelativistic-ion

\footnotetext{
${ }^{\text {a) }}$ Author to whom correspondence should be addressed; electronic mail: pkroy@lbl.gov

${ }^{b)}$ Current address: Stanford Research Systems, Inc., 1290-D Reamwood Ave. Sunnyvale, CA 94089.

${ }^{c)}$ Current address: San Onofre Nuclear Generating (SONGS) Mesa, G48B, P.O. Box 128, San Clemente, CA 92672
}

beams where electric fields predominate. We have developed for this purpose a scanning electron-beam probe, which has negligible interaction with the ion beam, and which does not require physical motion of its source position. Other advantages of this diagnostic are: (1) good detection resolution, (2) negligible loss of energy or particles of electron or ion beams, (3) absence of thermal or radiation load on structure and (4) absence of secondary electron emission during ion beam investigation. Though electron beam probes have been suggested and built, ${ }^{12-14}$ including use of the commercial black and white emittance-dominated television gun, there were difficulties in instrumentation and measurement techniques; lack of a low energy electron-detecting scintillator with sufficient photon emission; difficulty of experimental setup; methods of data extraction; and including inaccurate placement and measurement of the e-beam on axis due to deflection by low-level background magnetic fields. The techniques described in this article have overcome many of the difficulties encountered in earlier approaches. Instead of physically translating the electron gun, ${ }^{13}$ dipole magnets in a chicane are used, allowing the electron beam to sweep over the ion beam without a change in their direction through the ion beam. The e-beam emittance growth, due to space charge and geometrical aberration, is minimized by choosing a gun of a low current $(1 \mu \mathrm{A})$ and a design based on calculations using the SIMION three-dimensional ray-tracing code. Low level background magnetic fields $(<1 \mathrm{G})$ are cancelled using a rectangular shape Helmholtz coil around the e-beam. In this system, a $5-8 \mathrm{keV}$ e-beam, from a fixed location, is transversely swept across an ion beam by two dipole magnets but maintaining its horizontal direction towards an electron detecting scintillator. The image of the e-beam on the scintillator is monitored using a computerized gated camera, and data are extracted and analyzed by a program written using the image analysis program IMAGE-J. ${ }^{15}$ 


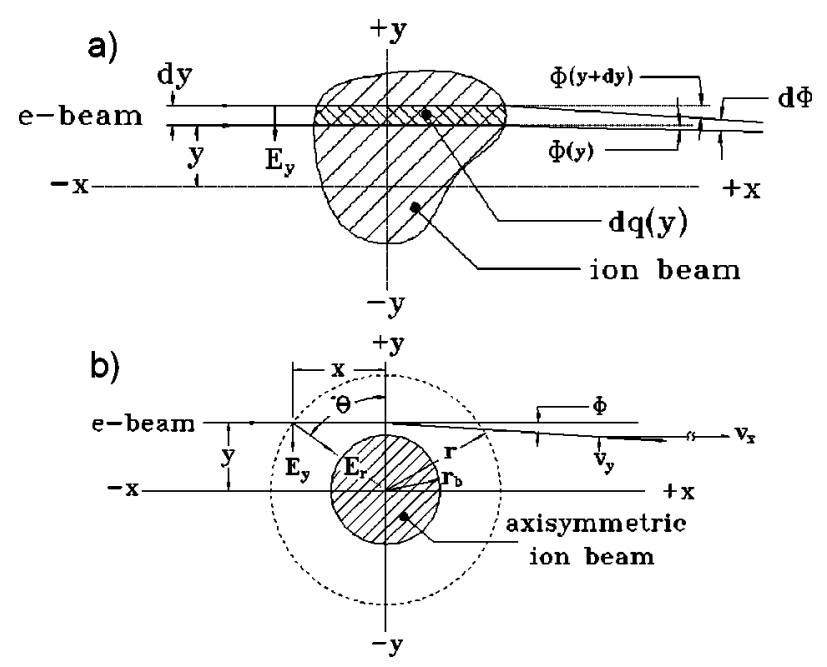

FIG. 1. (a) A transverse cross-sectional view of an arbitrary charge distribution in the $X$ and $Y$ directions. Probe electrons of velocity $v_{x}$ are directed horizontally from left to right $(-X$ to $+X)$ at various heights, $Y$, and acquire a velocity $v_{y}$ orthogonal to $v_{x}$, (b) an axisymmetric nonrelativistic charged particle beam which is long relative to its transverse dimensions, and where the charge density is a function of radius only, the electric field $E_{r}$ at any given radial distance, $r$, from the beam center will be proportional to the charge enclosed within a concentric cylindrical Gaussian surface of radius $r$, as shown by the dashed circle.

The conceptual basis of the diagnostic is presented in Sec. II; the design and development of the system are presented in Sec. III. Characterization of the mechanical construction, electron-beam transport, and its trajectory across the magnets is presented in Sec. IV. Ion beamline results are presented in Sec. V of this article.

\section{CONCEPTUAL BASIS OF THE DIAGNOSTIC}

Assume a collection of charged particles having an arbitrary two-dimensional (2D) density distribution which is invariant along the third longitudinal direction. This is descriptive of the NTX ion and other long beams. We direct a beam of probe electrons transversely through the ion beam. By measuring transverse deflections of probe electrons orthogonal to their direction of motion after they pass through an ion beam, the charge distribution of the ion beam can be calculated as described in the next paragraph. To determine an arbitrary 2D transverse distribution, tomographic techniques using more than one direction of the electron beam are required, which is presently beyond the scope of this experiment. Nevertheless, the following theory is easily and straightforwardly extendible for determining the Radon Transform of the 2D density profile, which is the basis of tomographic reconstruction.

Figure 1(a) shows a transverse cross-sectional view of an arbitrary charge distribution in the $X$ and $Y$ directions that is very long and invariant in the $Z$ direction (not shown in the figure). Probe electrons of velocity $v_{x}$ are directed horizontally from left to right $(-X$ to $+X)$ at various heights, $Y$, and acquire a velocity $v_{y}$ orthogonal to $v_{x}$. For nonrelativistic beams of this nature we can assume the electron moves in such a way that $v_{x} \gg v_{y}$ and thus the trajectories can be considered straight lines with a small angular deflection after passing near the ion beam. The e-beam accelerates when approaching the ion beam, and decelerates when moving away from the ion beam, however the net velocity change in $X$ is zero, and the variation is very small, less than $1 \%$, typically, and thus $v_{x}$ can be considered constant along the e-beam trajectory. Furthermore, we ignore any longitudinal (Z) electric field, as it does not contribute to transverse deflection.

The deflection angle can be obtained from the equation of motion

$$
\frac{d v_{y}}{d t}=-\frac{q_{e}}{m} E_{y},
$$

where $q_{e}$ and $m$ are the charge and mass of the electron, and $E_{y}$ is the electric field produced by the ion beam charge distribution on the $x-y$ plane.

Since $v_{x}=d x / d t$, we have

$$
\begin{aligned}
& \frac{d v_{y}}{d x}=-\frac{q_{e}}{m v_{x}} E_{y}, \text { and } \\
& \frac{d \Phi}{d x}=-\frac{q_{e}}{m v_{x}^{2}} E_{y},
\end{aligned}
$$

where $\Phi=v_{y} / v_{x}$. Therefore, the deflection angle is given by integrating $E_{y}$ along the e-beam trajectory

$$
\Phi=-\frac{q_{e}}{m v_{x}^{2}} \int E_{y} d x .
$$

From Maxwell's equation $\nabla \cdot \mathbf{E}=\rho / \varepsilon_{0}$, and since we ignore $E_{z}$,

$$
\frac{\partial E_{x}}{\partial x}+\frac{\partial E_{y}}{\partial y}=\rho(x, y) / \varepsilon_{0} .
$$

Integrating this equation along an e-beam trajectory $(y$ =constant) we have

$$
\int \frac{\partial E_{x}}{\partial x} d x+\int \frac{\partial E_{y}}{\partial y} d x=1 / \varepsilon_{0} \int \rho(x, y) d x .
$$

Here, the first term is zero since $E_{x}$ always integrates to zero (from $X=-\infty$ to $X=+\infty$ ). If we define the charge intercepted by a thin wire or a slit at position $y$ and of width $d y$ as $d q(y)$, we have

$$
\frac{d}{d y} \int E_{y} d x \propto d q(y)
$$

and, from the relationship between the deflection angle $\Phi$ and the $y$ component of the electric field $E_{y}$, we have

$$
d q(y) \propto \frac{d \Phi}{d y} .
$$

Therefore a slit or wire-scan charge profile measurement can be related to an e-beam deflection scan measurement through its derivative. To determine, without making any assumptions of charge distribution shape, the $2 \mathrm{D}$ charge density distribution, a full set of deflection measurements over many different electron-beam directions $(X)$ and offsets, $(Y)$ must be made. Doing so produces the Radon Transform of the 2D density distribution. Once this is obtained, various 




FIG. 2. A schematic of the nonintercepting electron-beam diagnostic system consists of four dipole magnets (D1-D4), an electron gun (e-gun), and electron-beam imaging YAP scintillator (detector, S). The e-beam travels parallel to its unperturbed axis in the gap of magnets D2 and D3, by the field effects of magnets D1 and D2. Again, by the effect of magnets D3 and D4 the e-beam trajectory converge back matches along the horizontal axis at the detector $(\mathrm{S})$, when there is no ion beam.

methods of computed tomography can be used to invert the Radon Transform, yielding the density distribution.

The above method of analysis simplifies when the beam density distribution is axisymmetric. Analytic solutions for various axisymmetric one-dimensional (1D) charge distribution functions can be formulated. Figure 1(b) shows an axisymmetric nonrelativistic charged particle beam which is long relative to its transverse dimensions, and where the charge density is a function of radius only, the electric field $E_{r}$ at any given radial distance, $r$, from the beam center will be proportional to the charge enclosed within a concentric cylindrical Gaussian surface of radius $r$, as shown by the dashed circle. For an electron beam passing through an ion beam, the amount of line charge density "seen" by the e-beam will vary as the e-beam travels through. This results in a decrease of the acquired transverse velocity, which has a functional dependence on the e-beam offset $y$ from the ion beam axis, going to zero for zero offset $y$. By scanning the e-beam through various offsets $y$, the radial dependence of $\lambda$ (and thus the volumetric charge density $\rho$ ) can be calculated. As an example, for an exponentially decaying density distribution where

$$
\rho(r)=\rho_{0} e^{-r / r_{0}}
$$

vertical aquired velocity $v_{y}$ is

$$
v_{y} \propto \int_{-\pi / 2}^{\pi / 2} 1-e^{-y / r_{0} \sec (\theta)} d \theta,
$$

where $r_{0}$ is the exponential decay radius. Similar formulas have been derived for hard-edged uniform, Gaussian, and Gaussian-hollow axisymmetric beam density distributions. Common to all solutions is the result that deflection angle is invariant with $Y$, once the entire charge distribution is not intercepted. The above analyses assume linear electron trajectories from $X=-\infty X=+\infty$ with a ground potential at infinity. The actual diagnostic is compact, with ground surfaces near the beam with a complicated electron trajectory. Figure 2 shows a typical electron-beam trajectory of the diagnostic system, and is described in detail in the next paragraph. $\mathrm{Nu}-$ merical simulation must be used to determine axisymmetric charge density distribution for this complicated geometry. Figure 3 shows numerical calculations of an $8 \mathrm{keV}$ electronbeam deflection for a typical NTX, $\mathrm{K}^{+}$ion beam of radius $1 \mathrm{~cm}$, energy of $264 \mathrm{keV}$, with a current of $25 \mathrm{~mA}$, assuming a Gaussian axis symmetric charge density.

\section{DESIGN AND DEVELOPMENT}

Figure 2 shows the e-beam diagnostic system consists of an electron gun (e gun), the e-beam image detector, and four dipole magnets (D1-D4) of equal strengths for a chicane system, wired in series to produce the field directions shown. The idea of the magnetic chicane system is that the e-beam trajectory can be swept in the vertical direction, while remaining parallel to the horizontal axis in the gap between the magnets D2 and D3. In the absence of an ion beam, D1 and D2 generate a parallel offset of the e-beam trajectory, and D3 and D4 return the e-beam to its initial direction. Now, if an ion beam is drifting perpendicularly to the e-beam in the space between the magnets D2 and D3, the e-beam will be deflected by the electrostatic charge of the ion beam, and its trajectory through magnets D3 and D4 is altered. By measuring the net deflection on a scintillator (S), as a function of the e-beam scan height or offset, the profile of the ion beam is characterized. The offset of the electron beam as it enters the beam is determined by the strength of the magnets, and the final e-beam deflection is measured as a function of the initial e-beam offset.

Development of the diagnostic includes: (1) selection of a compact low emittance electron gun to provide a small

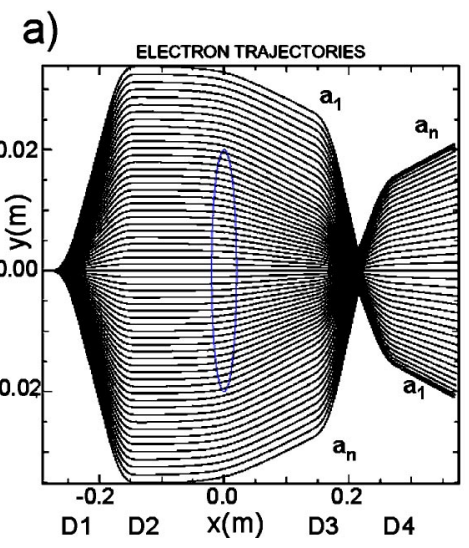

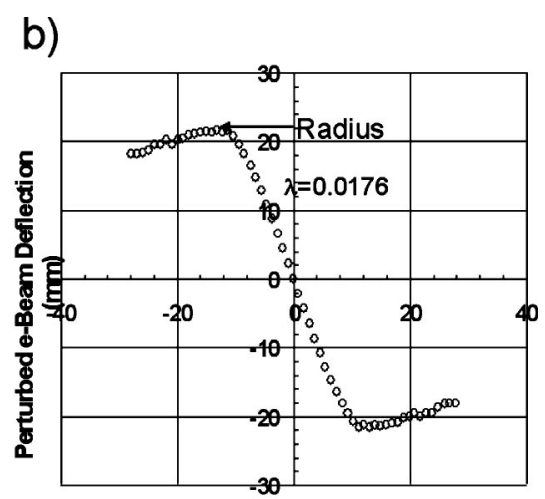

E-Beam Scan Height (mm)
FIG. 3. (Color online) Numerical calculations of electron-beam deflection (a) in the vicinity of an ion beam using four dipole magnets D1 to D4. The positions of the magnets are shown as indicated in axial scale, (b) calculated perturbed e-beam by an ion beam as a function of e-beam scan height. The ion beam radius, energy, and current were $1 \mathrm{~cm}, 264 \mathrm{keV}$, and $25 \mathrm{~mA}$, respectively, for an $8 \mathrm{keV}$ e-beam. 


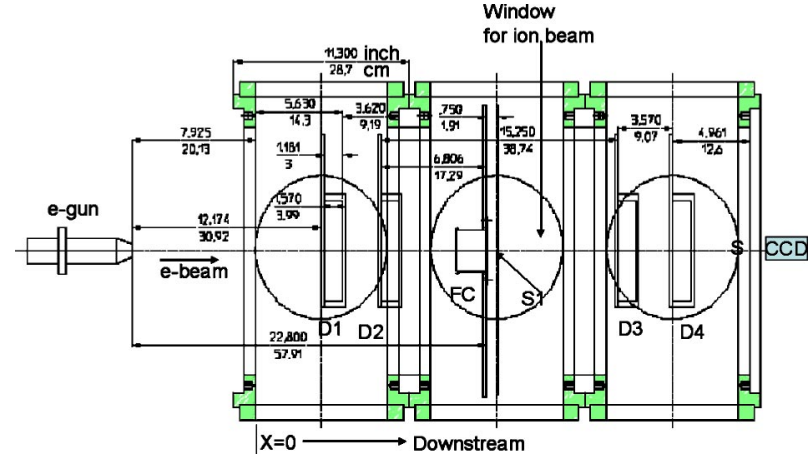

FIG. 4. (Color online) A schematic of the diagnostic with an electron gun (e-gun), dipole magnets (D1-D4), scintillator (S), vertical drive removable scintillator (S1), removable Faraday cup (FC), and a CCD camera associated with a computer (PC) have been installed outside of the chamber.

beam spot for good resolution. The gun needs to be compact to minimize space requirements and for easy handling, and the cathode heating has to be used without additional heat shielding and cooling systems. (2) Selection of a scintillator for detection of low energy electron beams. The scintillator was specially prepared in order to receive a usable optical signal by removing e-beam charges to ground and thus to minimize noise of electrons near the detector surface. (3) Design, construction and assembly of chicane magnets, and (4) design and construction of magnetic shielding hardware (Helmholtz coil in our setup), to cancel ambient magnetic field around the diagnostic chamber. Figure 4 shows an initial sketch of assembly. A brief description of each of these major components is presented below.

\section{A. Electron gun}

A Kimball Physics electron gun, model EGG-3101, steptuned, $0-10 \mathrm{kV}, 0-10 \mu \mathrm{A}$ electron current using a $\mathrm{LaB}_{6}$ cathode $^{16,17}$ was used. The gun has a thermal energy spread $(\Delta E)$ of $\sim 4 \mathrm{eV}$ with a Gaussian beam profile, and $\pm 0.01 \% / \mathrm{h}$ beam stability. The gun has the option of pulse lengths ranging from $50 \mathrm{~ns}$ to $1 \mathrm{~ms}$ with repetition rates up to $20 \%$ duty cycle, which will allow time-resolved measurements of the ion beam from head to tail.

\section{B. Chicane magnets}

The chicane magnets provide a vertical scan (translation) of the e-beam over $\pm 3 \mathrm{~cm}$. A magnetic induction range of $B= \pm 43.5 \mathrm{G}$ is required in each magnet gap to provide this translation range. It was desirable to minimize current requirements for a low-cost implementation compatible with high vacuum. Four identical compact iron-dominated, dc normal-conductor $\mathrm{H}$-shaped dipole magnets were designed and verified for field quality using the PANDIRA code. ${ }^{18}$ Figure 5 shows various quarter-section flux plots. For a missteered beam not passing along the magnet midline or for e-beam widths having a cross section comparable to the magnet half gap, there could be an undesirable transverse steering effect from the longitudinal fringe field component $B_{z}$ which could lead to e-beam loss. This component will have a nonzero cross product with the e-beam vertical velocity $(Y)$ component that is present between magnets D1 and
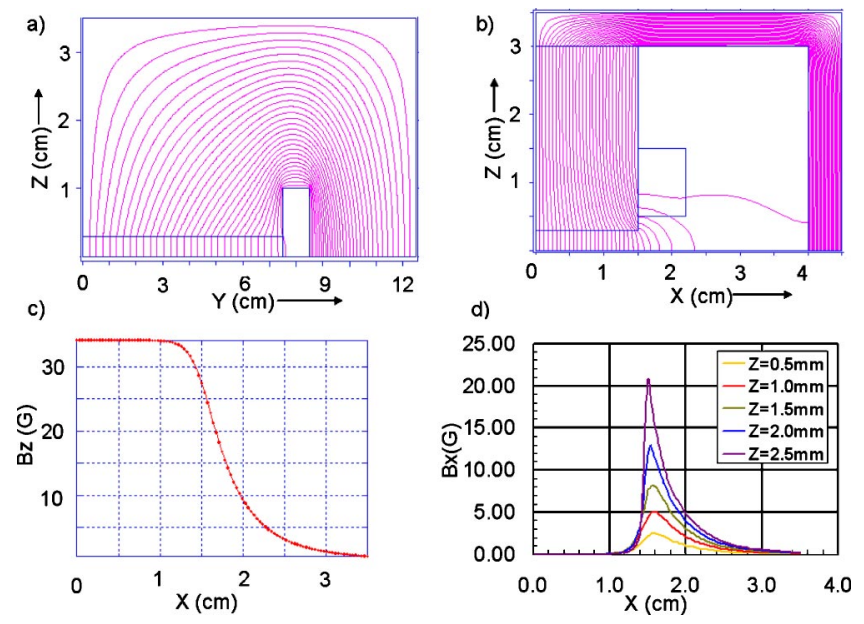

FIG. 5. (Color online). Quarter section flux plots in the (a) transverse $(X$ $-Y)$, (b) longitudinal $(X-Z)$ planes, (c) midplane gap field $\left(B_{z}\right)$ corresponding to (b), and (d) axial fringe field component $B_{x}$ for various e-beam trajectories offset from the magnet midplane, when $Z \neq 0$ in (b).

D2 and between the magnets D3 and D4 (Fig. 2). The program $\mathrm{SF}^{18}$ was used in conjunction with PANDIRA to generate the axial fringe field along the e-beam direction for several different values of the (local $Y$ ) distance from the midline, as shown in the Fig. 5(d).

The dipoles are iron dominated to minimize current and resistive heat loss, provide high field uniformity and to minimize stray magnetic field. They are designed to operate in vacuum, in order to eliminate the need for a vacuum chamber that can fit between the pole tips. Aluminum coils were machined from 6061-T6 aluminum, then hard anodized for electrical insulation, eliminating the need for organic or fiber based inorganic insulation, which would be less vacuum compatible. A low current density minimizes resistive loss, and allows coil cooling by radiation only, eliminating the need for water cooling in vacuum. A small number of turns minimizes voltage drop and conductor total area. Table I shows parameters of a chicane magnet. Figure 6(a) shows an exploded view of the dipole magnet, Fig. 6(b) shows the mechanical construction of a magnet following the exploded view, Fig. 6(c) shows the side view of two magnets assembled into the diagnostic chamber, and Fig. 6(d) shows the electrical circuit of the magnets. Leads are gold plated to minimize contact resistance. Annealed ultralow-carbon steel was used for its high permeability and low outgassing. The calculated current includes fringe field that provides additional vertical bending beyond the pole-tip edge as seen in Fig. 5(c) at $X=1.5 \mathrm{~cm}$.

TABLE I. Chicane magnet parameters.
Pole-tip gap

Pole-tip height

Horizontal drift distance between magnets

Required current to achieve an e-beam height of $40 \mathrm{~mm}$ (32 $\mathrm{G}$ for $5 \mathrm{keV}$ )
Magnet length along e-beam
$30 \mathrm{~mm}$

$6 \mathrm{~mm}$

$150 \mathrm{~mm}$

$60 \mathrm{~mm}$

$6.7 \mathrm{~A}$ at $3 \mathrm{mV} / \mathrm{coil}$ 
a)

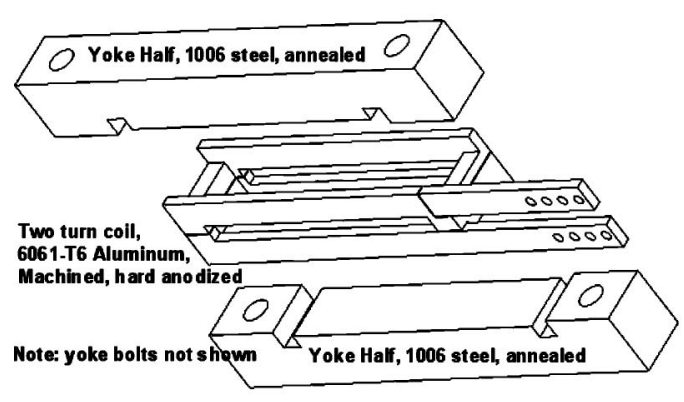

c)

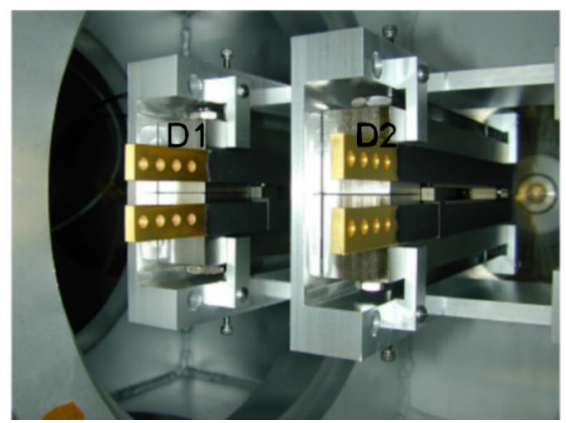

b)

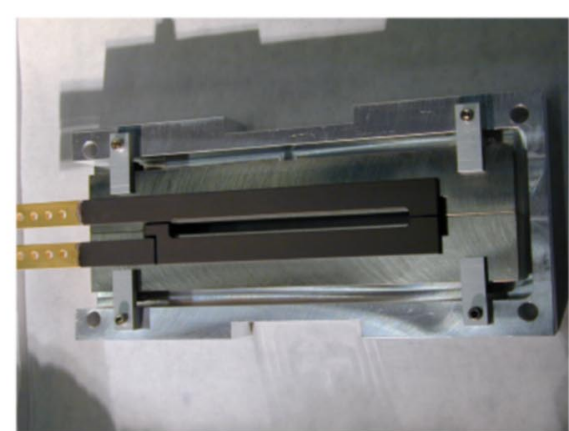

d)

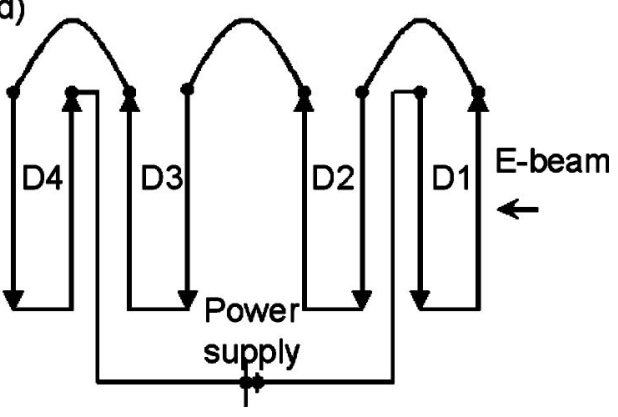

FIG. 6. (Color online). Dipole magnet: (a) an exploded view of the dipole magnet; (b) the mechanical construction of a magnet, (c) the side view of two magnets assembled into the diagnostic chamber, and (d) the electrical circuit loop of the magnets.

\section{Scintillator}

There has been significant development of the scintillator as particle an optics detecting detector in the past. ${ }^{19,20}$ Yttrium-aluminum-perovskite scintillator (YAP: $\mathrm{Ce})^{21-24}$ was used in this system for electron-beam detection. Table II shows data of the YAP: Ce scintillator. The stopping depth of the $5 \mathrm{keV}$ e-beams in the YAP scintillator was calculated to be $0.028 \mu \mathrm{m}$ using the Bethe formula. ${ }^{25,26}$ If a segment or pixel of the scintillator is isolated from the ground, charge can build up on the front surface of the scintillator which may repel the incoming beam particles as they approach the scintillator surface. A $100 \mathrm{~nm}$ aluminum coating was evaporated on the scintillator to drain this charge. The capacitance of scintillator-embedded charge relative to the aluminum layer is calculated to be $5.28 \times 10^{-7} \mathrm{~F}$. A $R C$ time constant $2.23 \times 10^{-13} \mathrm{~s}$ was calculated for the surface coating, and is sufficient for charge draining. This thickness of aluminum has a stopping power of approximately $1.2 \mathrm{keV}$.

\section{Geomagnetic field cancellation}

The earth's geomagnetic field (primarily the vertical component) acts to deflect the electron beam transversely. The average vertical geomagnetic field is nominally $0.44 \mathrm{G}$ at Lawrence Berkeley National Laboratory (LBNL); it com-

\section{TABLE II. YAP: Ce data.}

\begin{tabular}{lc}
\hline \hline & \\
Density & $5.57 \mathrm{~g} / \mathrm{cm}^{3}$ \\
Maximum emission length & $350 \mathrm{~nm}$ \\
Decay time & $27 \mathrm{~ns}$ \\
Photon number emission ability & $18 / \mathrm{keV}$ \\
Thermal expansion & $4-11 \mathrm{ppm}$ \\
Melting point & $1875^{\circ} \mathrm{C}$ \\
\hline \hline
\end{tabular}

bines with the steel building and the presence of large steel shielding plates produces a vertical field as high as $0.7 \mathrm{G}$. Without correction, this field would produce a sagitta of $1 \mathrm{~cm}$ over a drift distance of $0.5 \mathrm{~m}$, precluding the e-beam from passing through the chicane magnet system. The e-beam has a tolerance of approx $\pm 1 \mathrm{~mm}$ transverse displacement, thus requiring at least tenfold reduction in the vertical geomagnetic field component. A modified aspect ratio Helmholtz coil was designed to cancel this vertical field component. A rectangular shape was chosen for compactness and ease of fabrication. The dimensions are $1.5 \mathrm{~m}$ length (along e-beam) $\times 0.75 \mathrm{~m}$ width $\times 0.75 \mathrm{~m}$ height. The top and bottom coils each have ten turns of No. 12AWG insulated copper conductor, and are connected in series. Approximately $70 \mathrm{~A}$ turns per coil are needed to produce a $0.7 \mathrm{G}$ central field. Figure 7 shows a quadrant field plot in the plane transverse to the e-beam direction, with 70 A turns of current in a single filament (units are $\mathrm{cm}$ ). The end legs of the coils increase the field somewhat at the ends; however, the e-beam path is located largely within the central " $2 \mathrm{D}$ " portion of the coil. The maximum vertical excursion of the e-beam is $4 \mathrm{~cm}$

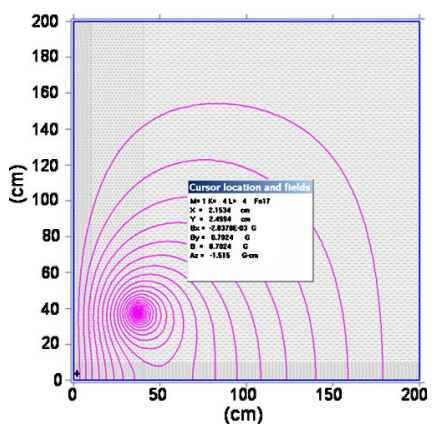

FIG. 7. (Color online) Plot of Helmholtz coil field (1/4 section). 


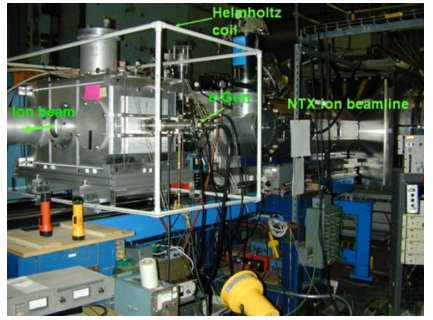

FIG. 8. (Color online). Electron-beam diagnostic, as installed in the NTX ion beamline. The diagnostic is surrounded by a rectangular frame shaped Helmholtz coil. Electron beam from the e-gun perpendicularly crosses the NTX ion beam. A detector (scintillator) to detect e-beam spot is located at the other side of the e-gun system.

(in $Y$ ) which is still in the good field region of the Helmholtz coil.

\section{MECHANICAL CONSTRUCTION}

The locations of the e-gun, dipole magnets (D1-D4) and scintillator (S) are shown in Fig. 4, and a picture of the installation, surrounded by a Helmholtz coil in the NTX beamline, is shown in Fig. 8. A computer-controlled, imageintensified charge-coupled device (CCD) camera imaged the e-beam spot on the scintillator. The pressure of the system was $\leqslant 10^{-7}$ Torr. Using a current of $4 \mathrm{~A}$ in the Helmholtz coil, the e-beam was directed in a straight line along the axis. Figure 9 shows measurements of the e-beam trajectory. The lines with solid circles and diamonds represent e-beam trajectories with and without energizing the Helmholtz coil field, respectively. Using the Helmholtz coil field, it was observed that the e-beam was placed on the axis. Without Helmholtz coil field, the e-beam became off axis. The e-beam size varies with e-beam current and energy. A 1-mm-diam beam spot size was measured for an $8 \mathrm{keV}$, $1 \mu \mathrm{A}$ e-beam at a distance of $1 \mathrm{~m}$. We were able to change the beamlet size by changing the e-beam current.

A bipolar power supply was connected in series with the dipole magnets as shown earlier in Fig. 6(d) operated with a current range of $\pm 8 \mathrm{~A}$, thus providing a wide range of magnetic field. The electron-beam trajectory sweeping height from its axis, at an ion beam path, as a function of dipole magnet current, is calculated to be

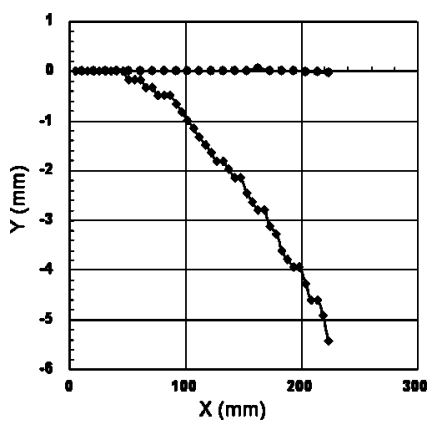

FIG. 9. Electron-beam trajectory along its axis. The lines with solid circles and diamonds represent measurements of e-beam trajectory with and without Helmholtz coil, respectively. The initial measuring point $x=0$ is at the wall, closer to the e gun, of the diagnostic box which is at a distance of $39.69 \mathrm{~cm}$ from the scintillator S1 (Fig. 4). The measurements only span part of the experimental region between the $x=0$ and Scintillator $\mathrm{S} 1$.

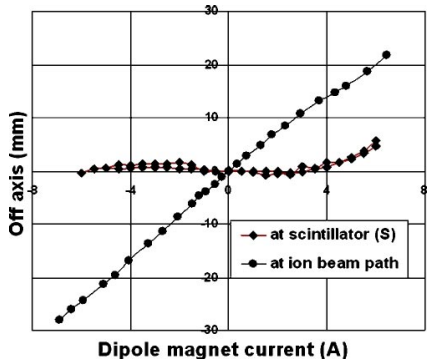

FIG. 10. Measurements of e-beam height from its axis vs current through dipole magnets. The line with circles shows e-beam trajectory displacement at the ion beam path (between magnets D2 and D3 in Fig. 2), and the line with diamonds shows the e-beam converging on the horizontal line at the scintillator (S) after passing through all four magnets (D1-D4).

$$
h(B)=2\{r(B)[1-\cos \Phi(B)]+L[\tan \Phi(B)]\},
$$

where $r(B)$ is radius of electron motion, usually defined by $m v / q B$ with the usual meaning of the symbols, $L$ is the magnet length along e-beam, $\Phi$ is the angle of deflection, defined as $\sin ^{-1}(L / r)$, and field, $B$, is defined by

$$
B=\frac{I \eta \xi N \mu_{0}}{g},
$$

where $I$ is the current in the dipole magnet, $\eta$ is the magnetic efficiency (0.961), $\xi$ is the fringes field factor (1), $N$ is the number of turns (2), constant term, $\mu_{0}=4 \pi \times 10^{-7}$ in $\mathrm{Tm} / \mathrm{A}$, and $g$ is the gap of a magnet $(0.006 \mathrm{~m})$.

Figure 10 shows measurements of the deflection height of the e-beam at two different locations for the same magnet current. The lines with circles and diamonds show measurements of the e-beam trajectory height at the ion beam path (between magnets D2 and D3 in the Fig. 2), and converging at the horizontal line (e-beam propagating axis) at the scintillator (S) after it passed through all four magnets (D1-D4), respectively. We measured a displacement of $\pm 3 \mathrm{~cm}$ by applying a current of $\pm 7 \mathrm{~A}$, and this indicated that a large, 6 -cm-diam, ion beam cross-section scan is possible using the diagnostic. The e-beam was converging with the horizontal axis at the scintillator (S) after traveling through the magnets D3 and D4 (Fig. 2), when there was no ion beam traveling through the gap between the magnets D2 and D3. In Fig. 10, measured data agreed well with our diagnostic design concept as magnets D1 and D2 generated a parallel offset of the e-beam trajectory and D3 and D4 returned to its initial condition in the absence of an ion beam. In the data, some residual vertical deflection remaining at the end of the e-beam line was observed. One possible cause for this residual vertical deflection is that there is a time-dependent magnetic field hysteresis. Another possibility is that the gap fields of the four magnets are not exactly equal in magnitude.

We have studied two different modes for operating the chicane system. The first is to place the scintillator at the focal point after D4. The second is to place the scintillator at around the position of D3 (with D3 and D4 removed). The first option involves a smaller scintillator, but a longer electron path, and hence greater susceptibility to unwanted e-beam perturbation by stray fields. The second option requires a larger scintillator, but the e-beam trajectory is shorter, and measurement of the beam deflection due to ion 
a)

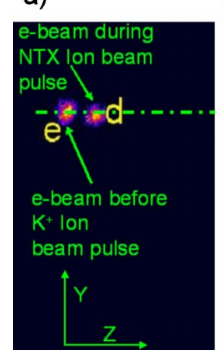

b)

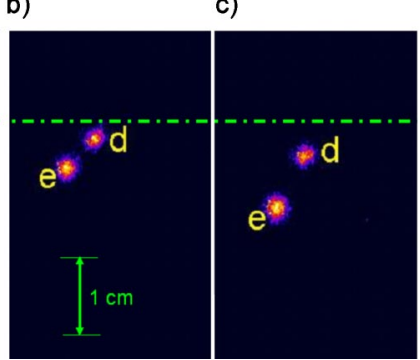

d)

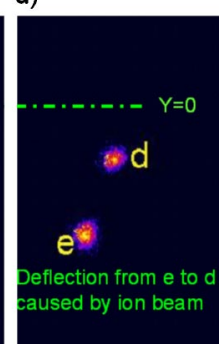

FIG. 11. (Color online). Deflected and undeflected electron beams on the scintillator at four different scan heights. Ion beam centerline is at $y=0$ in each frame. For this measurement, we removed magnets D3 and D4 to allow freer passage of the e-beam. In each frame, the left hand spot (e) is the undeflected e-beam; its vertical position is the scan height. The right hand spot (d) is a time exposure of the e-beam spot traversing the central $6 \mu \mathrm{s}$ of the $10-\mu$ s-long ion beam.

charge is more direct. During the later stage of the experiment, we procured a larger scintillator and made measurements in the second configuration with two magnets (D1 and D2) only, and D3 and D4 were removed. Most of the beam data presented in this report were taken in this configuration.

\section{RESULTS OF ION BEAM PROFILE MEASUREMENTS}

The diagnostic system was installed in the NTX ion beam line, as shown in the Fig. 8, to measure the ion beam profile. The measured profile was compared with a direct scintillator measurement for the same ion beam parameters.

Using a current of $4.4 \mathrm{~A}$ across the Helmholtz coil, the $8 \mathrm{keV}$ e-beam was directed in a straight line towards the scintillator. A sweeping height of $\pm 25 \mathrm{~mm}$ of e-beam trajectory was measured by a gap field using a current of $\pm 6.5 \mathrm{~A}(B= \pm 26.0 \mathrm{G}$ in magnet gaps) along the ion beam path. A displacement of $\pm 3.2 \mathrm{~cm}$ was obtained by applying a current $\pm 8 \mathrm{~A}$. The ion beamline was obtained from our present ongoing NTX experiments. This ion beamline consists of three major sections: a potassium source chamber, a 2.4-m-long magnetic transport section with four pulsed quadrupoles, and a 1-m-long neutralization drift section. Details of the ion beamline are presented elsewhere. ${ }^{27-29}$ The diagnostic was installed in the $1 \mathrm{~m}$ drift section. The steady e-beam was directed perpendicular to the ion beam axis.

Figure 11 shows both deflected and undeflected electron beams on the scintillator at four different scan heights $y$. Ion beam centerline is at $y=0$ in each frame. For this measurement, we removed magnets D3 and D4 to allow freer passage of the e-beam. In each frame, the left hand spot (e) is the undeflected e-beam; its vertical position is the scan height. The right hand spot (d) is a time exposure of the e-beam spot traversing the central $6 \mu$ s of the 10- $\mu$ s-long ion beam.

The radial field of the ion beam pulls the e-beam towards beam center. A complete set of measurements of e-beam deflection for scan heights $-22 \mathrm{~mm}<Y<19 \mathrm{~mm}$ was made in a different set of experiments. Figure 12 shows the transverse displacement of the e-beam trajectory by the ion beam as the e-beam scanning height was varied relative to its axis to scan the ion beam radial profile. The lines with solid diamonds

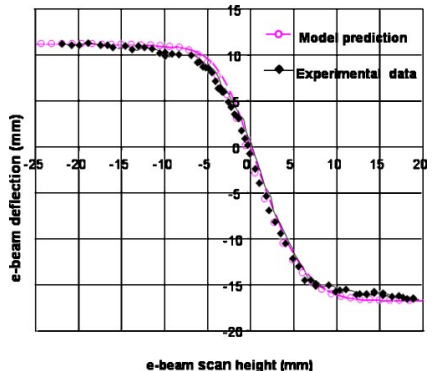

FIG. 12. (Color online) Transverse displacement of perturbed e-beam trajectory by an ion beam as a function of unperturbed e-beam trajectory. The lines with solid diamonds and hollow circles show, respectively, experimental data and model predictions. For the experimental data, the e-beam was vertically translated across the ion beam. The e-beam locations on the scintillator, S, were documented before and during the ion beam pulse. The difference between these two measurements was the result of the e-beam trajectory perturbation by the ion beam for a height of the e-beam. A Python tracking code was used to model the e-beam deflection assuming an axisymmetric Gaussian charge density distribution of $14 \mathrm{~mm}$ width and $\lambda=2.0$ $\times 10^{-8} \mathrm{C} / \mathrm{m}$ for $264 \mathrm{keV}$, e-beam energy of $8 \mathrm{keV}$ and $2 \mathrm{D}$ slab geometry. From the initial conditions, the accelerations were calculated as the e beam passed over the magnetic and electric fields and the new position and velocity were updated at every time step. At the end of the trajectory, the final deflection was obtained.

and hollow circles show, respectively, experimental data and theoretical predictions. For the experimental data, the e-beam was vertically translated over a $4 \mathrm{~cm}$ height across the ion beam. The e-beam locations on the scintillator, $\mathrm{S}$, were documented before and during the ion beam pulse. The difference between these two measurements was the result of the e-beam trajectory perturbation by the ion beam. A Python tracking code was used to model the e-beam deflection assuming an axissymmetric Gaussian charge density distribution of $14 \mathrm{~mm}$ width and $\lambda=2.0 \times 10^{-8} \mathrm{C} / \mathrm{m}$, e-beam energy of $8 \mathrm{keV}$ and 2D $(z, x)$ slab geometry. From the initial conditions, the accelerations were calculated as the e-beam passed over the magnetic and electric fields and the new position and velocity were updated at every time step. At the end of the trajectory, the final deflection was obtained. Varying the $B$ field yielded different impact parameters. The experimental data agree with the model prediction for a particular set of parameters describing the charge density distribution.

Figure 13 shows a comparison of the integrated ion beam profile measured optically by placing a ceramic scintillator, 98\% alumina, in front of the ion beam, and by this

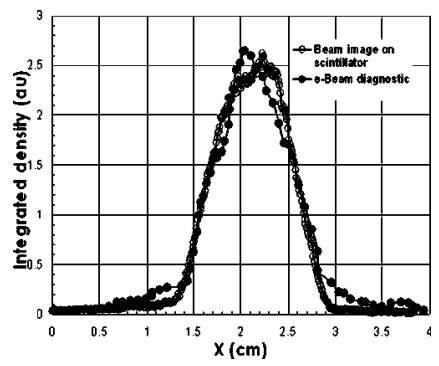

FIG. 13. A comparison of the integrated ion beam profile measurements. The line with hollow and solid circles shows, respectively, ion beam profile measured destructively (directly placing a ceramic scintillator in front of the ion beam), and nondestructively using the e-beam diagnostic system. 
e-beam system. The integrated density was obtained by doing a least-square fit on the data presented in Fig. 12 using Eq. (3). The derived profiles agreed well for this $1.3 \mathrm{~cm}$ ion beam profile. Because of relative insensitivity to background subtraction, inherent in the optical imaging, the e-beam diagnostic has an advantage over the direct measurement of the beam profile with the scintillator. This is important when measuring beam halos.

We have successfully designed, constructed and characterized an e-beam diagnostic system, installed it in the NTX ion beam line, and used it to both validate the approach and measure the ion beam profile. The e-beam deflection by the ion beam was also monitored for a long exposure, up to $15 \mu$ s time delay, to observe the response of the e-beam with respect to the ion beam head and tail. Though the vertical perturbation is constant during the beam pulse, the axial position slightly varied with time delay. It is inferred that during the passage of the ion beam head and tail, some of the e-beam particles might be deflected axially. It appears to indicate a longitudinal electric field that grows during the beam pulse. Also, the e-beam deflection was measurable for a time window as short as $10 \mathrm{~ns}$, which is the smallest time window available with this camera. This diagnostic is useful for a range of ion beam conditions including those associated with the NTX plasma neutralized ion beam experiments. We have developed and demonstrated an electron beam-based diagnostic system for measuring a transverse 1D charge distribution of an ion beam. This concept can be expanded with tomographic techniques to measure the 2D charge distribution.

\section{ACKNOWLEDGMENTS}

The authors are grateful to Dr. G. Logan, Dr. Christine Celata, Dr. Alex Friedman and all scientific members and technical staff of the Heavy Ion Fusion Virtual National Laboratory (HIF-VNL) for their support of research in this area. The authors thank Patrick O'shea, Rami Alfred Kishek and Santiago Bernal of the University of Maryland for design guidelines for the Helmholtz coil. This work was supported by the Office of Science. Fusion Energy Sciences, U.S. Department of Energy under Contract No. DE-AC03$76 \mathrm{SF} 00098$.

\footnotetext{
${ }^{1}$ R. O. Bangerter, Nucl. Instrum. Methods Phys. Res. A 464, 17 (2001).

${ }^{2}$ S. S. Yu, A. Anders, S. Eylon, E. Henestroza, P. K. Roy, D. Shuman, W. Waldron, W. Sharp, D. Rose, D. Welch, P. Efthimion, and E. Gilson, in Proceedings of the 2003 Particle Accelerator Conference, edited by J.
}

Chew (IEEE, New York, 2003), p. 98.

${ }^{3}$ E. Henestroza, A. Andres, P. K. Roy, W. Sharp, E. Shmuel, S. S. Yu, D. Rose, D. Welch, P. Efthimion, and E. Gilson, in Ref. 2, p. 2622.

${ }^{4}$ P. A. Seidl, D. Baca, F. M. Bieniosek, A. Faltens, S. M. Lund, A. W. Molvik, L. R. Prost, and W. L. Waldron, Laser Part. Beams 20, 435 (2002).

${ }^{5}$ D. Baca, J. W. Kwan, J. K. Wu, and C. Golcher, in Ref. 2, p. 3294.

${ }^{6}$ S. Eylon, E. Henestroza, P. K. Roy, and S. S. Yu, in Ref. 2, p. 2616.

${ }^{7}$ C. Lejeune and J. Aubert, Applied Charged Particle Optics, edited by A. Septier (Academic, San Diego, 1980), Part A, p. 9.

${ }^{8}$ J. G. Wang, D. X. Wang, and M. Reiser, Nucl. Instrum. Methods Phys. Res. A 307, 190 (1991)

${ }^{9}$ P. V. Logatchov, P. A. Bak, A. A. Starostenko, N. S. Dikansky, V. S. Tupikov, K. V. Gubin, V. M. Mishnev, M. B. Korabelnikov, and M. G. Fedotov, Proceedings Particle Acclerator Conference, New York City, 29 March to 2 April 1999, p. 2167.

${ }^{10}$ A. A. Starostenko, P. A. Bak, Ye. A. Gusev, N. S. Dikansky, P. V. Logatchov, and A. R. Frolov, EPAC 2000, Vienna, 26-30 June 2000, p. 1720 .

${ }^{11}$ P. V. Logatchov, P. A. Bak, A. A. Starostenko, N. S. Dikansky, V. S. Tupikov, K. V. Gubin, V. M. Mishnev, M. B. Korabelnikov, and M. G. Fedotov, in Proceedings of the 1999 Particle Accelerator Conference, edited by A. Luccio and W. MacKay (IEEE, Piscataway, NJ, 1999), p. 2167.

${ }^{12}$ P. K. Roy, S. Eylon, R. Hannink, E. Henestroza, J. Ludvig, D. Shuman, and S. S. Yu, in Ref. 2, p. 2625.

${ }^{13}$ J. Shiloh, M. Lampel, and R. Sah, Rev. Sci. Instrum. 54, 46 (1983).

${ }^{14}$ T. Weis, R. Dolling, P. Gross, J. Pozimski, G. Riehl, J. Wiegand, and H. Klein, Part. Accel. 37,38, 2 (1992).

${ }^{15}$ M. D. Abramoff, P. J. Magelhaes, and S. J. Ram, Image Processing with ImageJ, Biophotonics International, Vol. 11, Issue 7, 2004, pp. 36-42, and http://rsb.info.nih.gov/ij/

${ }^{16}$ J. M. Lafferty, J. Appl. Phys. 22, 299 (1951).

${ }^{17}$ P. K. Roy et al., Rev. Sci. Instrum. 67, 4098 (1996).

${ }^{18}$ POISSON/SUPERFISH, LANL Accelerator code group, Vol. 6.28, LANL.

${ }^{19}$ R. Hofstadter, IEEE Trans. Nucl. Sci. NS-22, 13(1975).

${ }^{20}$ R. L. Heath, R. Hofstadter, and E. B. Hughes, Nucl. Instrum. Methods 162, 431 (1979).

${ }^{21}$ R. Autrata, P. Schauer, J. Kvapil, and J. Kvapil, Scanning 5, 91 (1983).

${ }^{22}$ S. E. Derenzo and W. W. Moses, Proc. CRYSTAL 2000, 125(1992); LBNL Report No. LBL-33295, 1992.

${ }^{23}$ S. E. Derenzo, M. J. Weber, W. W. Moses, and C. Dujardin, IEEE Trans. Nucl. Sci. NS-47, 860 (2000).

${ }^{24}$ Scintillator Crystal data, Marketech Int. Inc., Port Townsend, WA.

${ }^{25}$ www.aps.anl.gov/techpub/lsnotes/ls165/ls165/html

${ }^{26}$ Handbook of Accelerator Physics and Engineering, edited by A. W. Chao and M. Tigner (World Scientific, Singapore, 1999), p. 2.

${ }^{27}$ S. S. Yu, Bull. Am. Phys. Soc. 48, 333 (2003).

${ }^{28}$ E. Henestroza, S. Eylon, P. K. Roy, S. S. Yu, A. Anders, F. M. Bieniosek, W. G. Greenway, B. G. Logan, R. A. MacGill, D. B. Shuman, D. L. Vanecek, W. L. Waldron, W. M. Sharp, T. L. Houck, R. C. Davidson, P. C. Efthimion, E. P. Gilson, A. B. Sefkow, D. R. Welch, D. V. Rose, and C. L. Olson, Phys. Rev. ST Accel. Beams 7, 083501 (2004).

${ }^{29}$ P. K. Roy, S. S. Yu, S. Eylon, E. Henestroza, A. Anders, F. M. Bieniosek, W. G. Greenway, B. G. Logan, W. L. Waldron, D. L. Vanecek, D. R. Welch, D. V. Rose, R. C. Davidson, P. C. Efthimion, E. P. Gilson, A. B. Sefkow, and W. M. Sharp, Phys. Plasmas 11, 2890 (2004). 